\title{
Euclidean-valued group cohomology is always reduced
}

\author{
Tim Austin*
}

\begin{abstract}
Let $K$ be a topological field and $G$ a countable discrete group. Then for any linear $G$-action on a finite-dimensional vector space over $K$, the groups of coboundaries in the inhomogeneous bar resolution are closed in all degrees, and hence the cohomology is reduced in all degrees. This can be deduced from a more general automatic-closure theorem for continuous linear transformations between inverse limits of finite-dimensional vector spaces.
\end{abstract}

Keywords: Finite-dimensional representations; reduced cohomology; automatic closure

MSC (2010): 20J06, 20C99, 46A13

Let $G$ be a countable discrete group and $\pi: G \frown M$ a continuous action on a topological Abelian group. A fundamental tool for describing the structure of this $G$-module is the group cohomology $\mathrm{H}^{*}(G, M)$. This is often defined in terms of the inhomogeneous bar resolution

$$
M \stackrel{d_{1}}{\longrightarrow} \mathcal{C}^{1}(G, M) \stackrel{d_{2}}{\longrightarrow} \mathcal{C}^{2}(G, M) \stackrel{d_{3}}{\longrightarrow} \cdots,
$$

where $\mathcal{C}^{p}(G, M)$ is the group of all functions $G^{p} \longrightarrow M$, and $d_{p}: \mathcal{C}^{p-1}(G, M) \longrightarrow$ $\mathcal{C}^{p}(G, M)$ is the coboundary operator defined by

$$
\begin{array}{rl}
d_{p} f\left(g_{1}, \ldots, d_{p}\right):=\pi^{g_{1}} f\left(g_{2}, \ldots, g_{p}\right)+\sum_{i=1}^{p}(-1)^{i} & f\left(g_{1}, \ldots, g_{i} g_{i+1}, \ldots, g_{p}\right) \\
& +(-1)^{p-1} f\left(g_{1}, \ldots, g_{p-1}\right) .
\end{array}
$$

In terms of these objects, one sets

$$
\begin{aligned}
& \mathcal{Z}^{p}(G, M):=\operatorname{ker} d_{p+1}, \mathcal{B}^{p}(G, M):=\operatorname{img} d_{p}, \\
& \text { and } \mathrm{H}^{p}(G, M):=\mathcal{Z}^{p}(G, M) / \mathcal{B}^{p}(G, M)
\end{aligned}
$$

${ }^{*}$ Research supported by a fellowship from the Clay Mathematics Institute 
(where $d_{0}$ is interepreted as 0 ).

The group of cochains $\mathcal{C}^{p}(G, M)=M^{G^{p}}$ may naturally be endowed with the product topology arising from the topology of $M$. (Note that if $G$ is infinite and $p \geqslant 1$, then this will not be discrete even if $M$ is a discrete module.) For this topology, one sees easily that each map $d_{p}$ is continuous, so ker $d_{p}$ is closed. However, $\operatorname{img} d_{p}$ need not be: that is, there may be cocycles $G^{p} \longrightarrow M$ that can be approximated arbitrarily well by coboundaries, but are not coboundaries themselves.

In some applications to geometry and dynamics, the non-closure of img $d_{p}$ is itself an important feature of the action $\pi$. For this reason, one also defines the reduced cohomology groups by

$$
\mathrm{H}_{\text {red }}^{p}(G, M):=\mathcal{Z}^{p}(G, M) / \overline{\mathcal{B}^{p}(G, M)} .
$$

The cohomology of this group and module is reduced in degree $p$ if $\mathcal{B}^{p}(G, M)$ is closed, and hence $\mathrm{H}^{p}(G, M)=\mathrm{H}_{\text {red }}^{p}(G, M)$.

In applications of reduced cohomology, $M$ is most often an infinite-dimensional Banach or Fréchet space. Most obviously, reduced cohomology in degree one provides an important characterization of Property (T) ([3, Section 2.12 and Chapter $3]$ ) and some generalizations of it to other Banach modules ([2]). The purpose of this note is to prove that cohomology is always reduced for finite-dimensional vector-space modules. It will turn out that there is no extra difficulty in allowing vector spaces over arbitrary topological fields, including $\mathbb{R}, \mathbb{C}$, local fields, or any other field with its discrete topology.

Theorem 1. Let $K$ be a topological field. If $G$ is a countable discrete group and $V$ is a finite-dimensional $K$-linear representation of $G$, then $\mathcal{B}^{p}(G, V)$ is a closed subgroup of $\mathcal{Z}^{p}(G, V)$ for all $p \in \mathbb{N}$.

It will be clear from the proof that the same result holds for many other natural choices of resolution used to compute $\mathrm{H}^{*}$, such as the homogeneous bar resolution. Indeed, in some special cases the right choice of resolution gives an immediate proof of Theorem 11. Most simply, the conclusion is clear if there is a resolution of finite type, since then all the cohomology groups are finite-dimensional; for instance, this holds for any finite-dimensional representation of a group of type $\mathrm{FP}_{\infty}$ (see, for instance, [6, Sections VIII.4 and VIII.5]). But the general argument below reaches beyond such cases.

The proof of Theorem 1 is motivated by the analogous result for compact coefficient modules, which is very simple: if $M$ is a compact Abelian $G$-module, then $\mathcal{C}^{p}(G, M)=M^{G^{p}}$ is also compact for every $p$, and hence the continuous image $\mathcal{B}^{p}(G, M):=d_{p-1}\left(\mathcal{C}^{p-1}(G, M)\right)$ is necessarily closed. 
To adapt this argument, we will prove a similar automatic-closure result for images of certain topological vector spaces over $K$, which may be of interest in its own right. To formulate this, we begin with the following definition.

Definition 2. A pro-f.-.d. space over $K$ is a topological $K$-vector space $V$ which is an inverse limit of an inverse sequence

$$
\cdots \stackrel{q_{m}}{\longrightarrow} V_{m} \stackrel{q_{m-1}}{\longrightarrow} \cdots \stackrel{q_{1}}{\longrightarrow} V_{1}
$$

in which every $V_{i}$ is a finite-dimensional $K$-vector space (with the Hausdorff topology given by identifying with $K^{\operatorname{dim} V_{i}}$ ), every $q_{i}$ is $K$-linear, and where this inverse limit is given the inverse limit topology.

More concretely, this means that $V$ can be identified with the subgroup of those sequences $\left(v_{i}\right)_{i} \in \prod_{i} V_{i}$ which are consistent, in that $q_{i}\left(v_{i+1}\right)=v_{i}$ for every $i$. For this subgroup, addition and scalar multiplication are pointwise, as in $\prod_{i} V_{i}$, and the topology is restricted from the product topology on $\prod_{i} V_{i}$. We will often denote such an inverse sequence by $\left(V_{i}, q_{i}^{j}\right)_{j \geqslant i \geqslant 1}$, where $q_{i}^{j}:=q_{i} \circ q_{i+1} \circ \cdots \circ q_{j-1}$ if $j>i$ and $q_{i}^{i}:=\operatorname{id}_{V_{i}}$, and we will often denote a consistent sequence by $\lim _{i \leftarrow} \leftarrow v_{i}$. The inverse limit $V$ is always equipped with a consistent family of continuous, $K$-linear quotient maps $Q_{i}: V \longrightarrow V_{i}$.

Of course, any finite-dimensional $K$-vector space may be interpreted as a prof.-d. space by letting $V_{i}:=V$ and $q_{i}:=\operatorname{id}_{V_{i}}$ for every $i$.

The result at the heart of this paper is the following.

Theorem 3 (Automatic closure theorem). If $T: V \longrightarrow W$ is a continuous $K$ linear transformations of pro-f.- $d$. K-vector spaces, then its image is a closed subspace of $W$.

This will be proved following a sequence of lemmas.

Lemma 4. If $V$ is the pro-f.- $d$. space given by the inverse sequence $\left(V_{i}, q_{i}^{j}\right)_{j \geqslant i \geqslant 1}$, and $Q_{i}: V \longrightarrow V_{i}$ are the resulting quotient maps, then a vector subspace $W \leqslant V$ is closed if and only if

$$
W=\bigcap_{i \geqslant 1} Q_{i}^{-1}\left(Q_{i}(W)\right) .
$$

Proof. For each $i, Q_{i}(W)$ a subspace of the finite-dimensional space $V_{i}$, so it is closed. Therefore the continuous pre-images $Q_{i}^{-1}\left(Q_{i}(W)\right)$ are all closed, and hence so is their intersection.

On the other hand, if $W$ is closed and $v \in V \backslash W$, then $v$ has an open neighbourhood disjoint from $W$. By definition of the inverse-limit topology, this implies that there is some $i \geqslant 1$ for which $Q_{i}(v)$ has an open neighbourhood $U_{i}$ such that $Q_{i}^{-1}\left(U_{i}\right) \cap W=\varnothing$. This now implies $Q_{i}(v) \notin Q_{i}(W)$. 
Lemma 5. Suppose that $V$ and $W$ are pro-f.-d. spaces over $K$, obtained from the inverse sequences $\left(V_{i}, q_{i}^{j}\right)_{j \geqslant i \geqslant 1}$ and $\left(W_{i}, r_{i}^{j}\right)_{j \geqslant i \geqslant 1}$ respectively. Suppose also that $T: V \longrightarrow W$ is a continuous $K$-linear transformation. Then there are a sequence $i_{1} \leqslant i_{2} \leqslant \cdots$ of indices and a sequence of $K$-linear transformations $T_{j}: V_{i_{j}} \longrightarrow W_{j}$ for which the following diagram commutes:

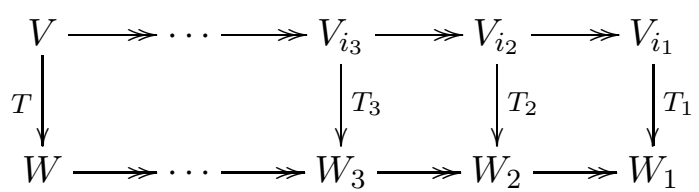

Proof. Let $Q_{i}: V \longrightarrow V_{i}$ and $R_{j}: W \longrightarrow W_{j}$ be the quotient maps.

If an $i_{j}$ and $T_{j}$ exist for some $j$, then the choice of $T_{j}$ for this $i_{j}$ is clearly unique. It therefore suffices to prove existence for any fixed $j$, since that uniqueness then forces the resulting family to be consistent, so that the above diagram commutes.

We must therefore show that, for each $j$, the composition $R_{j} \circ T: V \longrightarrow W_{j}$ factorizes through $Q_{i}$ for some $i$. Effectively, this restricts our attention to a finitedimensional target space $W$. After this restriction, it suffices to show that some $i$ satisfies $\operatorname{ker} Q_{i} \leqslant \operatorname{ker} T$.

Since ker $T$ is a closed vector subspace of $V$, the previous lemma gives

$$
\operatorname{ker} T=\bigcap_{i \geqslant 1} Q_{i}^{-1}\left(Q_{i}(\operatorname{ker} T)\right) \text {. }
$$

However, $\operatorname{ker} T$ has finite co-dimension in $V$, so this finite intersection must stabilize at some finite $i$, giving $\operatorname{ker} T=Q_{i}^{-1}\left(Q_{i}(\operatorname{ker} T)\right) \geqslant \operatorname{ker} Q_{i}$, as required.

In the setting of Lemma 5, the space $V$ may always be identified with the inverse limit of the cofinal inverse subsequence

$$
\cdots \stackrel{q_{i_{3}}^{i_{4}}}{\longrightarrow} V_{i_{3}} \stackrel{q_{i_{2}}^{i_{3}}}{\longrightarrow} V_{i_{2}} \stackrel{q_{i_{1}}^{i_{2}}}{\longrightarrow} V_{i_{1}} .
$$

Henceforth, after fixing a continuous linear transformation $T$ of interest, we will usually make this identification, and accordingly relabel this new inverse subsequence as $\left(V_{i}, q_{i}^{j}\right)_{j \geqslant i \geqslant 1}$.

Corollary 6. In the notation of Lemma 5 and after re-labeling as above,

$$
\operatorname{ker} T=\bigcap_{i \geqslant i} Q_{i}^{-1}\left(\operatorname{ker} T_{i}\right)
$$


In spite of this corollary, one can certainly have $\operatorname{ker} T_{i} \supsetneqq Q_{i}(\operatorname{ker} T)$ for all finite $i$. However, the following more subtle conclusion does always hold.

Lemma 7. With $T$ and its presentation as above, there is a non-decreasing function $\ell: \mathbb{N} \longrightarrow \mathbb{N}$ such that $\ell(i) \geqslant i$ and

$$
Q_{i}(\operatorname{ker} T)=q_{i}^{\ell}\left(\operatorname{ker} T_{\ell}\right) \quad \forall i \in \mathbb{N} \forall \ell \geqslant \ell(i) .
$$

Proof. For fixed $i,\left(q_{i}^{\ell}\left(\operatorname{ker} T_{\ell}\right)\right)_{\ell \geqslant i}$ is a decreasing sequence of subspaces of the finite-dimensional space $V_{i}$, hence must stabilize: that is, there is some $\ell \geqslant i$ such that

$$
q_{i}^{j}\left(\operatorname{ker} T_{j}\right)=q_{i}^{\ell}\left(\operatorname{ker} T_{\ell}\right) \quad \forall j \geqslant \ell .
$$

Let $\ell(i)$ be a choice of such an $\ell$, and assume without loss of generality that $\ell(i)>$ 1 . We will show that this common image is equal to $Q_{i}(\operatorname{ker} T)$. By re-labeling, it suffices to prove this when $i=1$.

Define a subsequence of indices $i_{0}<i_{1}<i_{2}<\ldots$ by letting $i_{0}:=1$ and recursively setting $i_{m+1}:=\ell\left(i_{m}\right)$.

Suppose that $v \in q_{1}^{i_{1}}\left(\operatorname{ker} T_{i_{1}}\right)$. By (1), $v$ also lies in $q_{1}^{i_{2}}\left(\operatorname{ker} T_{i_{2}}\right)$, since $i_{2}>$ $i_{1}$, so suppose $u \in \operatorname{ker} T_{i_{2}}$ satisfies $q_{1}^{i_{2}}(u)=v$, and now let $v_{1}:=q_{i_{1}}^{i_{2}}(u) \in$ $q_{i_{1}}^{i_{2}}\left(\operatorname{ker} T_{i_{2}}\right)$, so we still have $v=q_{1}^{i_{1}}\left(v_{1}\right)$.

Now, repeating this reasoning from (1) with $i_{1}$ in place of $i_{0}$ and $v_{1}$ in place of $v$, we obtain $v_{2} \in q_{i_{2}}^{i_{3}}\left(\operatorname{ker} T_{i_{3}}\right)$ such that $q_{i_{1}}^{i_{2}}\left(v_{2}\right)=v_{1}$. Continuing in this way gives a consistent sequence $\left(v_{j}\right)_{j \geqslant 1}$ in $\prod_{j \geqslant 1} V_{i_{j}}$ such that $v_{i_{j}} \in q_{i_{j}}^{i_{j+1}}\left(\operatorname{ker} T_{i_{j+1}}\right)$ for every $j$. Therefore $\lim _{i \leftarrow} v_{i} \operatorname{lies}$ in $\bigcap_{j \geqslant 1} Q_{i_{j}}^{-1}\left(\operatorname{ker} T_{i_{j}}\right)=\operatorname{ker} T$, and its image in $V_{1}$ is $v$, as required.

Proof of Theorem 3 Let $T: V \longrightarrow W$, and suppose that $w \in W$ is such that $w_{i}:=R_{i}(w) \in R_{i}(T(V))$ for every $i$. We need to show that $w \in T(V)$.

More explicitly, we need to find a consistent sequence $\left(v_{i}\right)_{i}$ in $\prod_{i} V_{i}$ such that $T_{i}\left(v_{i}\right)=w_{i}$ for each $i$. The sequence $\left(v_{i}\right)_{i}$ will be constructed recursively. The key idea is that we construct in parallel a sequence $\left(\tilde{v}_{i}\right)_{i} \in \prod_{i} V_{\ell(i)}$ such that $v_{i}=q_{i}^{\ell(i)}\left(\tilde{v}_{i}\right)$ for each $i$.

To begin, let $\tilde{v}_{1} \in V_{\ell(1)}$ be a $T_{\ell(1)}$-pre-image of $w_{\ell(1)}$, and let $v_{1}:=q_{1}^{\ell(1)}\left(\tilde{v}_{1}\right)$.

Now, suppose that for some $i \geqslant 2$ we have already constructed $\left(\tilde{v}_{j}\right)_{j=1}^{i-1} \in$ $\prod_{j=1}^{i-1} V_{\ell(j)}$ and $\left(v_{j}\right)_{j=1}^{i-1} \in \prod_{j=1}^{i-1} V_{j}$ such that

- $T_{\ell(j)}\left(\tilde{v}_{j}\right)=w_{\ell(j)}$,

- $v_{j}=q_{j}^{\ell(j)}\left(\tilde{v}_{j}\right)$, 
- and $q_{j^{\prime}}^{j}\left(v_{j}\right)=v_{j^{\prime}}$ whenever $j^{\prime} \leqslant j \leqslant i-1$ (note that we do not require the analog of this consistency for $\left.\left(\tilde{v}_{j}\right)_{j}\right)$.

We will construct $v_{i}$ and $\tilde{v}_{i}$ so that all of these properties still hold for the enlarged family.

To do this, let $v^{\prime}$ be any element of $T_{\ell(i)}^{-1}\left\{w_{\ell(i)}\right\}$. Then we have

$$
\begin{aligned}
& T_{\ell(i-1)} q_{\ell(i-1)}^{\ell(i)}\left(v^{\prime}\right)=r_{\ell(i-1)}^{\ell(i)} T_{\ell(i)}\left(v^{\prime}\right)=r_{\ell(i-1)}^{\ell(i)}\left(w_{\ell(i)}\right)=w_{\ell(i-1)}=T_{\ell(i-1)}\left(\tilde{v}_{i-1}\right) \\
& \Longrightarrow \quad \tilde{v}_{i-1}-q_{\ell(i-1)}^{\ell(i)}\left(v^{\prime}\right) \in \operatorname{ker} T_{\ell(i-1)} \\
& \Longrightarrow \quad q_{i-1}^{\ell(i-1)}\left(\tilde{v}_{i-1}\right)-q_{i-1}^{\ell(i)}\left(v^{\prime}\right)=v_{i-1}-q_{i-1}^{\ell(i)}\left(v^{\prime}\right) \in q_{i-1}^{\ell(i-1)}\left(\operatorname{ker} T_{\ell(i-1)}\right) .
\end{aligned}
$$

By the definition of $\ell(\cdot)$, this last subspace is equal to $q_{i-1}^{j}\left(\operatorname{ker} T_{j}\right)$ for any other $j \geqslant \ell(i-1)$; in particular, it is equal to $q_{i-1}^{\ell(i)}\left(\operatorname{ker} T_{\ell(i)}\right)$. Let $v^{\prime \prime} \in \operatorname{ker} T_{\ell(i)}$ be such that

$$
q_{i-1}^{\ell(i)}\left(v^{\prime \prime}\right)=v_{i-1}-q_{i-1}^{\ell(i)}\left(v^{\prime}\right) \quad \Longrightarrow \quad q_{i-1}^{\ell(i)}\left(v^{\prime}+v^{\prime \prime}\right)=v_{i-1} .
$$

Finally, letting $\tilde{v}_{i}:=v^{\prime}+v^{\prime \prime} \in V_{\ell(i)}$ and $v_{i}:=q_{i}^{\ell(i)}\left(\tilde{v}_{i}\right)$, one has

$$
T_{\ell(i)}\left(\tilde{v}_{i}\right)=T_{\ell(i)}\left(v^{\prime}+v^{\prime \prime}\right)=T_{\ell(i)}\left(v^{\prime}\right)=w_{\ell(i)}
$$

and

$$
q_{i-1}^{i}\left(v_{i}\right)=q_{i-1}^{\ell(i)}\left(v^{\prime}+v^{\prime \prime}\right)=v_{i-1},
$$

so the induction continues. This completes the proof.

Proof of Theorem 1 For each $p$, the group $\mathcal{C}^{p}(G, V)=V^{G^{p}}$ is a countable product of finite-dimensional $K$-vector-spaces, so it is a pro-f.-d. space. Since the boundary homomorphisms

$$
d_{p+1}: \mathcal{C}^{p}(G, V) \longrightarrow \mathcal{C}^{p+1}(G, V)
$$

are clearly continuous (as they are for cohomology with values in any topological module), by Theorem 3 they are also closed: that is,

$$
\mathcal{B}^{p}(G, V)=d_{p}\left(\mathcal{C}^{p-1}(G, V)\right)
$$

is closed in $\mathcal{Z}^{p}(G, V)$, as required.

Since a countable product of pro-f.-d. spaces with the product topology is still pro-f.-d., the above proof actually gives the following strengthening of Theorem 1 .

Corollary 8. If $G$ is a countable discrete group, $K$ is a topological field and $V$ is a pro-f.-d. $G$-space over $K$, then $\mathcal{B}^{p}(G, V)$ is closed in $\mathcal{Z}^{p}(G, V)$ for all $p \geqslant 0$. 
However, the above methods do not seem to bear on the following natural relative of Theorem 1 .

Question 9. Are there a countable group $G$ and an integer $p \geqslant 1$ such that the cohomology $\mathrm{H}^{p}(G, \mathbb{Z})$ is not reduced, where $\mathbb{Z}$ is given the trivial $G$-action? Can this occur for other discrete $G$-modules?

This was Question 7.4 in [1]. Of course, for $M$ with trivial $G$-action one always has $\mathcal{B}^{1}(G, M)=0$, so the interesting cases start at $p=2$. Moreover, whenever $M$ is a Polish $G$-module and one knows that $\mathrm{H}^{p}(G, M)$ is countable for some $p$, it follows that $\mathcal{B}^{p}(G, M)$ is closed for very abstract reasons. Indeed, $\mathcal{B}^{p}(G, M)=d_{p}\left(\mathcal{C}^{p-1}(G, M)\right)$ is a continuous image of a Polish space, hence analytic in the sense of Souslin. If $\mathcal{Z}^{p}(G, M) \backslash \mathcal{B}^{p}(G, M)$ is a countable union of cosets of $\mathcal{B}^{p}(G, M)$, then one has that $\mathcal{B}^{p}(G, M)$ is also co-analytic, hence Borel. As such it is either meagre in $\mathcal{Z}^{p}(G, M)$, or co-meagre inside some nonempty open set. Using again that countably many cosets of $\mathcal{B}^{p}(G, M)$ cover $\mathcal{Z}^{p}(G, M)$, it cannot be meager, so it is co-meager in some non-empty open subset $U \subseteq \mathcal{Z}^{p}(G, M)$. However, this now implies that

$$
\mathcal{B}^{p}(G, M) \supseteq\left(U \cap \mathcal{B}^{p}(G, M)\right)-\left(U \cap \mathcal{B}^{p}(G, M)\right),
$$

which contains a neighbourhood of 0 , so $\mathcal{B}^{p}(G, M)$ is open and therefore also closed.

This last argument applies, in particular, if $G$ satisfies some cohomological finiteness properties, such as $\mathrm{FP}_{k}$ for some $k>p$; see, for instance, [6, Chapter VIII].

One cannot hope to answer Question 9 positively using a method too close to that of the present note, because the analog of Theorem 3 is false for inverse limits of finitely-generated torsion-free Abelian groups.

Example 1. Let $W:=V:=\mathbb{Z}^{\mathbb{N}}$, the infinite product with the product topology, and let $T: V \longrightarrow W$ be given by

$$
T\left(p_{1}, p_{2}, p_{3}, \ldots\right):=\left(p_{1}-2 p_{2}, p_{2}-2 p_{3}, p_{3}-2 p_{4}, \ldots\right) .
$$

Each of $V$ and $W$ is an inverse limit for the sequence of projections $\mathbb{Z}^{n+1} \longrightarrow \mathbb{Z}^{n}$ onto the initial coordinates, and $T$ may be described using these projections via a commutative diagram as in Lemma 5, since each coordinate output by $T$ depends on only two input coordinates. It also follows that $T$ is continuous.

A point $\left(q_{1}, q_{2}, \ldots\right) \in \mathbb{Z}^{\mathbb{N}}$ lies in $T(V)$ if and only if there is some $\left(p_{1}, p_{2}, \ldots\right) \in$ $\mathbb{Z}^{\mathbb{N}}$ such that

$$
q_{1}=p_{1}-2 p_{2}, \quad q_{2}=p_{2}-2 p_{3}, \quad \ldots
$$


This re-arranges to give

$p_{2}=\frac{1}{2}\left(p_{1}-q_{1}\right), \quad p_{3}=\frac{1}{4}\left(p_{1}-q_{1}-2 q_{2}\right), \quad p_{4}=\frac{1}{8}\left(p_{1}-q_{1}-2 q_{2}-4 q_{3}\right), \quad \ldots$,

and this requires, in particular, that

$$
p_{1} \in \bigcap_{i \geqslant 1}\left(q_{1}+2 q_{2}+\cdots+2^{i-1} q_{i}+2^{i} \mathbb{Z}\right) .
$$

If one can find such an integer $p_{1}$, then the equations above allow one to find suitable $p_{j}$ for all $j \geqslant 1$. Such a $p_{1}$ exists, for example, if $q_{i}=0$ for all large enough $i$, since in that case one can let $p_{1}:=\sum_{i \geqslant 1} 2^{i-1} q_{i}$. However, it does not exist for $\left(q_{1}, q_{2}, \ldots\right)=(1,0,1,0, \ldots)$, since then one has

$$
\min \left\{|p| \mid p \in q_{1}+2 q_{2}+\cdots+2^{i-1} q_{i}+2^{i} \mathbb{Z}\right\} \sim \frac{1}{3} 2^{i} \longrightarrow \infty \quad \text { as } i \longrightarrow \infty .
$$

Therefore the image $T(V)$ is dense in $W$, but not equal to the whole of $W$. $\triangleleft$

It could be interesting to try to adapt the idea behind Example 1 into an example in which $\mathcal{B}^{p}(G, \mathbb{Z})$ is not closed for some $G$ and $p$. I do not know how to do this, but simpler examples show that in the 'mixed' setting of actions on torsionfree Abelian Lie groups, the analog of Theorem 1 is itself false. The following is essentially copied from the example in [1, Lemma 7.7].

Example 2. Let $\alpha, \beta \in \mathbb{R}$ be irrational and rationally independent, let $M=\mathbb{R} \times \mathbb{Z}^{2}$, and give it the $\mathbb{Z}$-action generated by

$$
T(t, p, q):=(t+p \alpha+q \beta, p, q) .
$$

Then an easy calculation gives that $\mathcal{Z}^{1}(\mathbb{Z}, M) \cong \operatorname{Hom}(\mathbb{Z}, \mathbb{R})=\mathbb{R}$, and under this isomorphism $\mathcal{B}^{1}(\mathbb{Z}, M)$ is identified with the submodule $\mathbb{Z} \alpha+\mathbb{Z} \beta$, which is dense but not closed in $\mathbb{R}$.

Another obvious extension of Theorem 1 would be to non-discrete groups $G$. In that setting there are several cohomology theories for Polish $G$-modules that could be of interest, such as $\mathrm{H}_{\mathrm{cts}}^{*}$, defined using continuous cochains, and $\mathrm{H}_{\mathrm{m}}^{*}$, defined using measurable cochains. In case the coefficient module is a Fréchet space, it was shown in [1, Theorem A] that these theories coincide.

Question 10. If $G$ is a locally compact, second countable group, is it true that $\mathrm{H}_{\mathrm{cts}}^{p}(G, V)$ is reduced for every $p$ and every Euclidean $G$-space $V$ ? Is it true that $\mathrm{H}_{\mathrm{m}}^{p}(G, \mathbb{Z})$ is reduced for every $p$ ? 
The paper [1] offers a more complete overview of various cohomology theories in this setting, and a more thorough set of further references.

The issue of Question 10 is potentially important for calculational problems in which one wishes to use a version of the Lyndon-Hochschild-Serre spectral sequence. If $H \unlhd G \rightarrow K$ is a short exact sequence of locally compact, second countable groups, and $V$ is a Fréchet $G$-module, then in principle this spectral sequence gives

$$
\mathrm{H}_{\mathrm{cts}}^{p}\left(H, \mathrm{H}_{\mathrm{cts}}^{q}(K, V)\right) \Longrightarrow \mathrm{H}_{\mathrm{cts}}^{p+q}(G, V) .
$$

However, for this to make sense one must consider continuous cochains $H^{p} \longrightarrow$ $\mathrm{H}_{\text {cts }}^{q}(K, V)$ for the quotient topology on $\mathrm{H}_{\text {cts }}^{q}(K, V)$, and the calculation works out correctly only if that quotient topology is Hausdorff. For this reason, an affirmative answer to Question 10 could enlarge the known domain of applicability of this spectral sequence. This issue is discussed in more detail for continuous cohomology into Fréchet modules in [5, Chapter IX], and for the related setting of measurable cohomology into Polish modules in [7]. It also appears in [4], which uses a comparison between $\mathrm{H}_{\mathrm{cts}}^{*}$ and a kind of $L_{\text {loc }}^{p}$-cohomology to justify some cases of the spectral sequence.

Question 10 does not fall within the methods of the present paper, because if $V$ is Euclidean but $G$ is non-discrete then the groups of continuous cochains $\mathcal{C}_{\text {cts }}^{p}(G, V)$ are not pro-f.-d. in the topology of locally uniform convergence. If Question 10 has an affirmative answer, it will presumably require a more specific analysis of the closure properties of the coboundary operators in this setting. Indeed, the paper [1] already contains examples of such arguments: see [1, Theorem D], which answers both parts of Question 10 affirmatively in case $G$ is almost connected (that is, its identity component is co-compact). The arguments there use comparison results between different cohomology theories, the Gleason-Montgomery-Zippin theorem, and the Lyndon-Hochschild-Serre spectral sequence. I expect that similar tools can answer Question 10 affirmatively for totally disconnected groups, since these may be written as compact extensions of discrete groups, and we know the desired result for both of those classes separately (by [1] and the present paper, respectively). But the fully general case seems more subtle.

\section{Acknowledgement}

I am grateful to David Fisher for helpful discussions and suggestions, and to the anonymous referee for providing some additional context and references. 


\section{References}

[1] T. Austin and C. C. Moore. Continuity properties of measurable group cohomology. Math. Ann., 356(3):885-937, 2013.

[2] U. Bader, A. Furman, T. Gelander, and N. Monod. Property (T) and rigidity for actions on Banach spaces. Acta Math., 198(1):57-105, 2007.

[3] B. Bekka, P. de la Harpe, and A. Valette. Kazhdan's property (T), volume 11 of New Mathematical Monographs. Cambridge University Press, Cambridge, 2008 .

[4] P. Blanc. Sur la cohomologie continue des groupes localement compacts. Ann. Sci. École Norm. Sup. (4), 12(2):137-168, 1979.

[5] A. Borel and N. Wallach. Continuous cohomology, discrete subgroups, and representations of reductive groups, volume 67 of Mathematical Surveys and Monographs. American Mathematical Society, Providence, RI, second edition, 2000.

[6] K. S. Brown. Cohomology of groups, volume 87 of Graduate Texts in Mathematics. Springer-Verlag, New York, 1982.

[7] C. C. Moore. Group extensions and cohomology for locally compact groups. III. Trans. Amer. Math. Soc., 221(1):1-33, 1976.

Courant Institute of Mathematical Sciences, New York University, 251 MERCER ST, NEW YORK NY 10012, USA

Email: timecims.nyu.edu

URL: cims.nyu.edu/ ${ }^{\text {tim }}$ 